\title{
Plasma tetranectin as a potential clinical biomarker for epilepsy and correlation with clinical and social characteristics
}

\author{
Ekta Singh Dahiya ${ }^{\mathrm{a}, *}$, Man Mohan Mehndiratta ${ }^{\mathrm{b}, 1}$, Krishna Kolappa Pillai $^{\mathrm{a}}$ \\ a Department of Pharmacology, Faculty of Pharmacy, Jamia Hamdard (Hamdard University), Hamdard Nagar, New Delhi, 110062, India \\ ${ }^{\mathrm{b}}$ Department of Neurology, Govind Ballabh Pant Hospital, New Delhi, 110002, India
}

\section{A R T I C L E I N F O}

\section{Article history:}

Received 12 August 2016

Accepted 17 December 2016

Available online 9 January 2017

\section{Keywords:}

Tetranectin

Biomarker

Newly-diagnosed epilepsy

Drug-effective epilepsy

Drug-refractory epilepsy

\begin{abstract}
A B S T R A C T
Background: Tetranectin concentration has been identified as a biomarker of several types of metastatic and malignant cancers. The role of tetranectin has also be seen in some neurological disorders. We aimed to estimate the plasma tetranectin concentration in different groups of people with epilepsy (PWE) followed-up for a year. As a secondary objective, the clinical and social characteristics were also correlated with the tetranectin levels.

Methods: We enrolled 90 subjects grouped as Newly-diagnosed epilepsy (NDE), Drug-effective epilepsy (DEE), and Drug-refractory epilepsy (DRE) and an age-gender matched control group $(n=30)$. The plasma samples were collected thrice at the six-month interval and were analysed for the tetranectin concentration using S-ELISA.

Results: The mean plasma tetranectin levels at the baseline test were significantly lower for the DEE $(6.294 \pm 0.806)$ and DRE $(7.572 \pm 0.545)$ groups compared with control group $(9.71 \pm 0.628)$ but not the $\operatorname{NDE}$ group ( $8.651 \pm 0.859$ vs. $9.71 \pm 0.628 ; p>0.05$ ). On a year of follow-up, the tetranectin levels for the NDE group significantly decreased $(p<0.001)$ matching with that of the DEE and DRE group. Multivariate linear regression analysis showed that gender $(p=0.035)$ in the DRE group and seizure type $(p=0.040)$ and diet $(\mathrm{p}=0.046)$ for the NDE group were significantly correlated.

Conclusion: The plasma tetranectin level in PWE significantly decreased as the disease progressed irrespective of the stage of epilepsy. Thus, tetranectin could be considered as a potential progressive biomarker for epilepsy. The study outcome suggests further investigation for the possible link of tetranectin levels with clinical and social parameters.
\end{abstract}

(c) 2016 Published by Elsevier, a division of RELX India, Pvt. Ltd on behalf of Indian Epilepsy Society.

\section{Introduction}

Epilepsy is a neurological disorder characterised by chronic, recurrent, unpredictable occurrence of seizures which affects nearly 50 million of the world's population., ${ }^{1,2}$ Despite increasing global health awareness, the gap between the incidence and prevalence of epilepsy in developed and developing nations still exists. Highlighting this fact about $80 \%$ of the total global burden of epilepsy is in developing countries. ${ }^{3}$ India has a prevalence rate of 6-10 million cases of epilepsy per year with the incidence rate of $27.3 / 100,000$ per year. ${ }^{4,5}$

\footnotetext{
* Corresponding author. Present address: National Institute for Stroke and Applied Neurosciences (NISAN), Faculty of Health and Environmental Sciences, Auckland University of Technology, Auckland, 0627, New Zealand.

E-mail address: ektasinghdahiya@gmail.com (E.S. Dahiya).

${ }^{1}$ Present address: Department of Neurology, Janakpuri Super Speciality Hospital, New Delhi, 10058, India.
}

The biomarkers identified for epilepsy revolves around two major mechanisms, i.e. epileptogenicity and epileptogenesis. Epileptogenicity biomarker identifies the genetic and molecular modulations; whereas biomarker for epileptogenesis deals with the development and progression of the disease. ${ }^{6}$ Epilepsy with its complexity and different clinical types do not have a unique biomarker. Identification of a potential blood biomarker in people with epilepsy (PWE) could be an important diagnostic tool along with the other epileptic molecular, EEG and imaging biomarkers.

Tetranectin is a glycoprotein which is encoded by CLEC3B gene (C-type lectin domain family 3 , member B) in human. ${ }^{7}$ Its role has been perceived prominently in the regulation of fibrinolysis, tissue growth, and proteolytic processes; presented in a variety of cells including monocytes, neutrophils, fibroblasts and osteoblasts. ${ }^{7,8}$ As a biomarker, tetranectin has been found to be downregulated with the progression of metastatic cancer (oral cancer, lymph node cancer) as well as in solid malignant cancer (breast, colonic and ovarian cancer). ${ }^{9,10}$ Furthermore, studies have shown the role of tetranectin in various neurological disorders such as multiple 
sclerosis, Parkinson disease, Alzheimer's disease, and epilepsy. Particularly studies mentioned that the level of CSF tetranectin increases while serum tetranectin level decreases in people with epilepsy compared with the healthy control. ${ }^{11,12}$

The research for the precise physiological role of tetranectin in epilepsy is still under investigation. This exploratory study was aimed to estimate and correlate the plasma tetranectin concentration in different groups of PWE at different clinical stages followed up for a year. The secondary objective was to correlate the clinical and social parameters with the proposed biomarker.

\section{Materials and methods}

\subsection{Subjects and Ethics committee approval}

The study was designed as a cross-sectional study and carried out in the Department of Neurology, Out Patient Department (OPD) at the University Hospital (Govind Ballabh Pant Hospital), New Delhi, during July 2011-January 2013. A total of 300 people diagnosed with epilepsy were screened based on their type of seizures defined in the International League Against Epilepsy (ILAE) in 1981, clinical history, neurological examination, EEG, and imaging (CT scan and MRI). As per the inclusion and exclusion criteria, 90 patients were enrolled and classified into three groups namely, Newly-diagnosed epilepsy (NDE, $n=30$ ), Drug-effective epilepsy (DEE, $n=30$ ), and Drug-refractory epilepsy (DRE, $n=30$ ). People who had one unprovoked seizure with high risk of relapse within past three months were defined as the NDE group; those who were seizure free for $\geq$ three years while taking a single antiepileptic drug (AED) or combination to treat epilepsy were categorized as DEE group; and those in whom no seizure control was there since the last two months while having three AEDs were grouped as DRE group. An age, gender, and sample size-matched control group $(n=30)$ with no evidence of neurological disorders or any history of seizures or exposure to AEDs was recruited. The study protocol was approved by the Institutional Ethics Committee, Maulana Azad Medical College (MAMC) \& Associated Hospitals, New Delhi.

\subsection{Procedure}

Each selected subject and the parents/guardians in case of children were subjected to sign the Inform Consent Form. The individual subject's clinical and social characteristics were entered in the Subject History Sheet. Blood samples were collected in $2 \mathrm{ml}$ EDTA vacutainers at the initial baseline and then twice after every six months. Samples were processed by centrifuging for $10 \mathrm{~min}$ at $2000 \mathrm{~g}$ to collect the plasma. The plasma was aliquot and stored at $-80^{\circ} \mathrm{C}$ until the analysis was done. The plasma tetranectin concentration at different time points was determined using a Human CLEC3 B sandwich ELISA kit (Bio-medical Assay Co. Ltd, Beijing, China). The assay procedure was followed according to the user manual provided by the manufacturer. A dilution of 1:100 was prepared for plasma samples and optical density was read at $450 \mathrm{~nm}$ using UV Spectrophotometer (PerkinElmer Lambda-20 Double-beam UV-vis spectrophotometer). The analysis was carried out at the Neurobehavioral Pharmacology Lab, Faculty of Pharmacy, Department of Pharmacology, Jamia Hamdard (Hamdard University), New Delhi, India.

\subsection{Statistical analysis}

The plasma tetranectin concentrations were expressed as mean \pm SD. The clinical and social characteristics of the study subjects were compiled as percentages. Differences between the variables from the three groups were assessed using one-way
Table 1

Clinical characteristics of the three groups of people with epilepsy.

\begin{tabular}{|c|c|c|c|}
\hline \multicolumn{4}{|l|}{ Clinical characteristics } \\
\hline & NDE & DEE & DRE \\
\hline \multicolumn{4}{|l|}{ Age, yrs } \\
\hline Mean & 19.8 & 24.97 & 23.2 \\
\hline Range & $6-52$ & $13-46$ & $7-45$ \\
\hline \multicolumn{4}{|l|}{ Sex, n (\%) } \\
\hline Male & $24(80.00)$ & $19(63.33)$ & $27(90.00)$ \\
\hline Female & $6(20.00)$ & $11(36.66)$ & $3(10.00)$ \\
\hline \multicolumn{4}{|l|}{ Onset of seizures, $\mathrm{n}(\%)$} \\
\hline$\leq 3 \mathrm{mo}$ & $30(100.00)$ & 0 & 0 \\
\hline$\leq 5$ yrs & 0 & $17(56.66)$ & $1(3.33)$ \\
\hline $6-15$ yrs & 0 & $11(36.66)$ & $20(66.66)$ \\
\hline $16-30$ yrs & 0 & $2(6.66)$ & $10(33.33)$ \\
\hline \multicolumn{4}{|l|}{ Family history, n (\%) } \\
\hline Yes & $7(23.33)$ & $4(13.33)$ & $5(16.66)$ \\
\hline No & $23(76.66)$ & $26(86.66)$ & $25(83.33)$ \\
\hline \multicolumn{4}{|l|}{ Frequency of seizures, $\mathrm{n}(\%)$} \\
\hline $1-2 / \mathrm{mo}$ & $30(100.00)$ & 0 & $11(36.66)$ \\
\hline $3-5 / \mathrm{mo}$ & 0 & 0 & $19(63.33)$ \\
\hline $1-2 / y r s$ & 0 & 0 & 0 \\
\hline $1 / 3-4$ yrs & 0 & $19(63.33)$ & 0 \\
\hline $1 / 5-10 \mathrm{yrs}$ & 0 & $11(36.66)$ & 0 \\
\hline \multicolumn{4}{|l|}{ Last episode, n (\%) } \\
\hline$<1-2$ mo & $30(100.00)$ & 0 & $30(100.00)$ \\
\hline$<1-5$ yrs & 0 & $18(60.00)$ & 0 \\
\hline$<6-10$ yrs & 0 & $12(40.00)$ & 0 \\
\hline \multicolumn{4}{|l|}{ Types of seizures, $\mathrm{n}(\%)$} \\
\hline GTCS & $17(56.66)$ & $19(63.33)$ & $11(36.66)$ \\
\hline Complex partial seizures & $6(20.00)$ & $4(13.33)$ & $5(16.66)$ \\
\hline Seizure with $2^{\circ}$ generalization & $6(20.00)$ & $5(16.66)$ & $10(33.33)$ \\
\hline Multiple seizures & $1(3.33)$ & $2(6.66)$ & $5(16.66)$ \\
\hline \multicolumn{4}{|l|}{ AEDs history, $\mathrm{n}$} \\
\hline 1 & 8 & 13 & 18 \\
\hline 2 & 13 & 9 & 24 \\
\hline 3 & 10 & 9 & 9 \\
\hline 4 & 1 & 2 & 4 \\
\hline 5 & 1 & 0 & 9 \\
\hline 6 & 0 & 1 & 2 \\
\hline 7 & 0 & 0 & 2 \\
\hline 8 & 0 & 0 & 1 \\
\hline
\end{tabular}

1-VPA, 2-CBZ, 3-PHT, 4-PB, 5- CB, 6- LTG, 7- LVT, 8- TP. NDE: Newly-diagnosed epilepsy, DEE: Drug-effective epilepsy, DRE: Drug-resistant epilepsy, mo: Months, yrs: Years.

ANOVA followed by Tukey-Kramer Multiple Comparison post hoc test. A multivariate linear regression was executed to identify the correlation of the factors with the biomarker level. The GraphPad Instat version 3.0 for Windows (GraphPad Software, San Diego, California, USA, www.graphpad.com) was used and a $\mathrm{p}$ value of $<0.05$ was considered as statistically significant.

\section{Results}

\subsection{Clinical and social characteristics}

Table 1 shows the summarised clinical characteristics. The mean age of the study population among the three groups was 22.65 years. The age range for the NDE group was $6-52$ years, the DEE group 13-46 years, and for the DRE group 7-45 years. The sex ratio varies from group to group basis, on the one hand, the DEE group had more male subjects than female (63.33\% vs. $36.66 \%$ ), whereas, both the DRE and NDE groups had only $10 \%$ and $20 \%$ of the female subjects included, respectively. A fair role of family history was found with a percentage of $17.77 \%$. The NDE group had GTCS with $56.66 \%$ as the most common type of seizure; seizures with secondary generalisation and complex partial seizures shared $20 \%$ each. For the DEE group, the most common type of seizure was GTCS (63.33\%) followed by seizures with secondary generalisation and complex partial seizures. A mixed ratio for the DRE group was there; GTCS and seizures with secondary generalisation shared the 
same percentage (36.66\% and 33.33\%), trailed by complex partial seizures, and multiple seizures with $16.66 \%$. The AEDs history implied that valproic acid (VPA), carbamazepine (CBZ), and phenytoin (PHT) were the drug of choice as monotherapy; whereas, clobazam $(\mathrm{CB})>$ phenobarbitone $(\mathrm{PB})>$ lamotrigine (LTG) > levetiracetam (LVT) were used as adjuvants for polytherapy regimen in the DRE group.

The social characteristics are presented in Table 2 . The majority of the study population were either had an education below 10th grade (NDE: $70.00 \%$, DEE: $43.33 \%$, and DRE: $53.33 \%$ ) or illiterate (NDE: $10.00 \%$, DEE: 6.66\%, and DRE: $30.00 \%$ ). A higher percentage of subjects belong to student category in the NDE group (56.66\%) whereas, in the DRE group, 60\% of the population were not working. A relation with the marital status was found with the OPD reporting, about $3 / 4$ th of the studied population was single and only $1 / 4$ th of those were married.

\subsection{Plasma tetranectin concentrations}

The mean plasma concentrations of tetranectin in three tests are shown in Table 3. The mean concentrations of the NDE group (8.651 \pm 0.859$)$ was comparable to the control group $(9.71 \pm 0.628)$, whereas concentrations of the DEE $(6.294 \pm 0.806)$ and DRE $(7.572 \pm 0.545)$ group were significantly decreased compared with the control group in the first test as baseline $(\mathrm{p}<0.001)$. At the first follow-up of six months, the plasma tetranectin level of the DRE group reduced further but the for the NDE group it remained more or less unchanged $(p>0.05)$. In the final test analysis at one-year follow-up, though the plasma tetranectin level of the DEE and DRE group remained unchanged to that in the previous tests; levels of the NDE group decreased significantly in comparison with the control group $(\mathrm{p}<0.001)$.

\subsection{Multivariate linear regression}

To establish the relationship between the tetranectin plasma concentration as a dependent variable and the predictor variables, we performed a multivariate linear regression model. The data from the baseline test (Test-I) was considered for each group. The tetranectin concentration being the dependent variable was

Table 2

Social characteristics of the three groups of people with epilepsy.

\begin{tabular}{|c|c|c|c|}
\hline \multicolumn{4}{|l|}{ Social characteristics } \\
\hline & NDE, n (\%) & DEE, n (\%) & DRE, n (\%) \\
\hline \multicolumn{4}{|l|}{ Education } \\
\hline$<10$ th & $21(70.00)$ & $13(43.33)$ & $16(53.33)$ \\
\hline 12th & $6(20.00)$ & $11(36.66)$ & $5(16.66)$ \\
\hline Graduate $\&$ above & 0 & $4(13.33)$ & 0 \\
\hline Illiterate & $3(10.00)$ & $2(6.66)$ & $9(30.00)$ \\
\hline \multicolumn{4}{|l|}{ Occupation } \\
\hline Service & $7(23.33)$ & $9(30.00)$ & $6(20.00)$ \\
\hline Business & 0 & 0 & 0 \\
\hline Self employed & $3(10.00)$ & $3(10.00)$ & 0 \\
\hline Student & $17(56.66)$ & $9(30.00)$ & $6(20.00)$ \\
\hline Not working & $3(10.00)$ & $9(30.00)$ & $18(60.00)$ \\
\hline \multicolumn{4}{|l|}{ Marital status } \\
\hline Single & $21(70.00)$ & $20(66.66)$ & $25(83.33)$ \\
\hline Married & $7(23.33)$ & $10(33.33)$ & $5(16.66)$ \\
\hline \multicolumn{4}{|l|}{ Personal habits } \\
\hline Drug abuse & 0 & 0 & 0 \\
\hline Alcoholic & $3(10.00)$ & $1(3.33)$ & $1(3.33)$ \\
\hline Smoking & $4(13.33)$ & $1(3.33)$ & $3(10.00)$ \\
\hline \multicolumn{4}{|l|}{ Dietary habits } \\
\hline Vegetarian & $9(30.00)$ & $11(36.66)$ & $9(30.00)$ \\
\hline Non-vegetarian & $21(70.00)$ & $19(63.33)$ & $21(70.00)$ \\
\hline
\end{tabular}

NDE: Newly-diagnosed epilepsy, DEE: Drug-effective epilepsy, DRE: Drug-resistant epilepsy.
Table 3

Plasma tetranectin concentration in three groups of people with epilepsy and control group.

\begin{tabular}{llll}
\hline Group & \multicolumn{3}{l}{ Tetranectin concentration $(\mu \mathrm{g} / \mathrm{ml})$} \\
& mean \pm S.D & & \\
\cline { 2 - 4 } & Test-1 & Test- & Test-3 \\
\hline Control & $9.71 \pm 0.628$ & $9.293 \pm 0.857$ & $9.293 \pm 0.857$ \\
NDE & $8.651 \pm 0.859$ ns & $8.726 \pm 0.822^{* * *}$ & $6.354 \pm 0.617^{* * *}$ \\
DEE & $6.294 \pm 0.806^{* * *}$ & $5.832 \pm 0.331^{* * *}$ & $6.068 \pm 0.776^{* * *}$ \\
DRE & $7.572 \pm 0.545^{* * *}$ & $5.394 \pm 0.580^{* * *}$ & $6.151 \pm 0.738^{* * *}$ \\
\hline
\end{tabular}

The values are shown as the mean $\pm \mathrm{SD},{ }^{*} \mathrm{p}<0.05,{ }^{* * *} \mathrm{p}<0.001$ compared to the Control. One-way ANOVA followed by Tukey-Kramer Multiple Comparison post hoc test. NDE: Newly-diagnosed epilepsy, DEE: Drug-effective epilepsy, DRE: Drugresistant epilepsy.

correlated with the clinical and social parameters. On comparing the three groups, the multivariate linear regression analysis revealed that overall these factors explained only the $16 \%$ to $21 \%$ of the total variability. Specifically, a significant correlation was seen for gender $(p=0.035)$ in the DRE group. Whereas, seizure type $(p=0.040)$ and diet $(p=0.046)$ were significantly correlated for the NDE group. On dummy coding moderation with regression analysis, it was evident that males had an inverse relation with the tetranectin under the drug-resistant group. On the other hand, based on the standardised coefficient $\beta$ people with NDE had GTCS as the most interacted followed by seizures with secondary generalisation, CPS and multiple seizures. Interestingly, the correlation of non-veg or veg diet could not be clearly defined provided with the same p-value of 0.021 .

\section{Discussion}

Our study confirmed that tetranectin has a defined role in the pathophysiology of epilepsy. The plasma tetranectin concentrations in PWE decreased significantly compared with the control group irrespective of the epilepsy state. These results are consistent with other published works. A previous study has stated that tetranectin is present in the normal brain and the CSF tetranectin level gets decreased in temporal lobe epilepsy. ${ }^{13}$ Another study reported that serum tetranectin level decreases while the CSF tetranectin increased in epilepsy and proposed that tetranectin could be a potential biomarker for epilepsy. ${ }^{11}$

Tetranectin is a kringle 4 protein binding plasminogen; the structure and its role in human physiology have been already worked out. There are study reports stating that tetranectin is present in neuronal cells, myelinated fibres of the white matters in both cerebrum and cerebellum, and CSF of the people with temporal lobe epilepsy (TLE), however, the exact role and mechanism of tetranectin in epileptogenesis are yet not clear. ${ }^{11,14}$

We found that at baseline sampling, the NDE group had comparable tetranectin level as that of healthy control subjects, whereas, the tetranectin levels in the DEE and DRE group were significantly downregulated. On a year of follow-up, the NDE group also showed a declined path similar to that of the DEE and DRE group. This suggests that as the disease advances the plasma tetranectin level decreases irrespective of the state of epilepsy. Furthermore, the Test-I was the baseline to compare the tetranectin level among the three groups and the control. The significant reduction in the tetranectin concentration points out its existing role in epilepsy. The subjects in the DEE and DRE groups had established history of epilepsy/seizures, in contrast to the NDE subjects at that stage. After a year of follow-up, fall of tetranectin level in the NDE group supported the initial hypothesis. However, the result regarding the DRE group was not in support of previously reported literature in the same context. ${ }^{11}$ According to that study, lower level of plasma tetranectin in the DRE group was correlated 
with the frequency of seizures. The same study showed that the NDE group showed a significant decrease compared with control. However, the subjects were sampled once and were not further followed up. These methodological differences could reflect the differences in result findings. As per our study findings, no difference at the six months follow-up but a significant reduction of tetranectin level in the NDE group after a year suggest that the biomarker levels are affected as the disease progress regardless of the stage of epilepsy.

The study has also highlighted the region-specific patterns amongst the people with epilepsy. During the screening of the study population, data for clinical and social characteristics was recorded. It turns out that there is a skewed gender ratio when it comes to reaching the hospital, as on an average nearly $77.77 \%$ of the study population constitute males. The family history of epilepsy among the study population was also evident. Although genetic testing was not performed, the subjects with known family history of epilepsy were counselled as per the case. GTCS being the most common type of seizure reported in the study agrees with other literature published. ${ }^{15,16}$ Besides the 1st generation AEDs (VPA, CBZ, and PHT) used as monotherapy, CB and PB were the drugs of choice as an adjuvant. Various clinical studies have reported LVT as safe and effective add-on drug in people with drugrefractory epilepsy. ${ }^{17,18}$ However, LVT was not commonly prescribed in the study population at our hospital settings. Being illiterate or having a just secondary education was correlated with unemployment among the PWE that directly associates with the different groups of epilepsy. More the frequency of seizures, more the patient, is cut off from the society; be it from education or the workplace. These findings were supported by other studies as well. ${ }^{19-21}$ Another factor that came into notice was the marital status; it turned out that higher proportion of the study subjects were single (75\%). The reason for that could be the fewer incidences of seizures occurs in higher ages of life, shifting of the female population after marriage, and societal and family pressure. The condition was worse with female subjects, for whom the stigma of having epilepsy hovers around them. This depicts the mental block of the society, even in the metro city like Delhi. This fact is not new in the country like India, others authors have also reported the same from different parts of the country. ${ }^{19-22}$ The tetranectin levels did not significantly correlate with the clinical and social parameters of the DEE subjects. However, the fact that there was a significant correlation in gender for DRE and type of seizure and diet for the NDE group pointed to dig deep into the concept. Whether these findings can be replicated in large study population or do a non-veg or veg diet would affect the progress of epilepsy in the NDE group.

The present exploratory study firmly concludes that plasma tetranectin level decreases in people with epilepsy as disease advances. Therefore, plasma tetranectin can be considered as a potential candidate for a biomarker of epilepsy that could assist the existing clinical biomarkers. However, with contrasting literature findings of no conclusive difference among the patients with refractory epilepsy per se, open up the future scope of the investigations. A larger sample size, total protein level analysis and prolonged follow-up of DRE and NDE subjects would overcome the limitation of this study. Even with a definite clinical association of tetranectin with epilepsy, further research is required to probe into the mechanism, role and diagnostic characteristic of tetranectin in epilepsy and other neurological disorders.

\section{Conflict of interest}

The authors have no conflict of interest.

\section{Acknowledgements}

The study was funded by the Council of Scientific and Industrial Research (CSIR), New Delhi, India (No. 09/591(0129)/2012EMRI). We would like to thanks the residents at the Neurology OPD at Govind Ballabh Pant Hospital, New Delhi for referring the people with epilepsy for screening.

\section{References}

1. Berg AT, Berkovic SF, Brodie MJ, Buchhalter J, Cross JH, van Emde Boas W, et al. Revised terminology and concepts for organization of seizures and epilepsies: report of the ILAE Commission on Classification and Terminology, 2005-2009. Epilepsia. 2010;51(4):676-685.

2. Joseph N, Kumar GS, Nelliyanil M. Pattern of seizure cases in tertiary care hospitals in Karnataka state of India. Ann Indian Acad Neurol. 2013;16(3):347.

3. De Boer HM, Mula M, Sander JW. The global burden and stigma of epilepsy. Epilepsy Behav. 2008;12(4):540-546.

4. Sridharan R, Murthy B. Prevalence and pattern of epilepsy in India. Epilepsia. 1999;40(5):631-636.

5. Bharucha NE. Epidemiology and treatment gap of epilepsy in India. Ann Indian Acad Neurol. 2012;15(4):352.

6. Engel J. Biomarkers in epilepsy: introduction. Biomark Med. 2011;5(5):537544.

7. Clemmensen I, Petersen LC, Kluft C. Purification and characterization of a novel, oligomeric, plasminogen kringle 4 binding protein from human plasma: tetranectin. Eur J Biochem. 1986;156(2):327-333.

8. Fuhlendorff J, Clemmensen I, Magnusson S. Primary structure of tetranectin, a plasminogen kringle 4 binding plasma protein: homology with asialoglycoprotein receptors and cartilage proteoglycan core protein. Biochemistry. 1987;26(21):6757-6764.

9. Arellano-Garcia ME, Li R, Liu X, Xie Y, Yan X, Loo JA, et al. Identification of tetranectin as a potential biomarker for metastatic oral cancer. Int J Mol Sci. 2010;11(9):3106-3121.

10. Høgdall CK. Human tetranectin: methodological and clinical studies. APMIS 1998;106(S86):7-31.

11. Wang L, Pan Y, Chen D, Xiao Z, Xi Z, Xiao F, et al. Tetranectin is a potential biomarker in cerebrospinal fluid and serum of patients with epilepsy. Clin Chim Acta. 2010;411(7):581-583.

12. Wang ES, Sun Y, Guo JG, Gao X, Hu JW, Zhou L, et al. Tetranectin and apolipoprotein A-I in cerebrospinal fluid as potential biomarkers for Parkinson's disease. Acta Neurol Scand. 2010;122(5):350-359.

13. Xiao F, Chen D, Lu Y, Xiao Z, Guan Lf, Yuan J, et al. Proteomic analysis of cerebrospinal fluid from patients with idiopathic temporal lobe epilepsy. Brain Res. 2009:1255:180-189.

14. Stoevring B, Jaliashvili I, Thougaard AV, Ensinger C, Høgdall CK, Rasmussen LS, et al. Tetranectin in cerebrospinal fluid: biochemical characterisation and evidence of intrathecal synthesis or selective uptake into CSF. Clin Chim Acta. 2005;359(1):65-71.

15. Mrabet H, Mrabet A, Zouari B, Ghachem R. Health-related quality of life of people with epilepsy compared with a general reference population: a Tunisian study. Epilepsia. 2004:45(7):838-843.

16. Rajabi F, Dabiran S, Hatmi ZN, Zamani G. Quality of life of Epileptic Patients Compared to General. Acta Med Iran. 2009;47(1):75-78.

17. Mbizvo GK, Dixon P, Hutton JL, Marson AG. Levetiracetam add-on for drugresistant focal epilepsy: an updated Cochrane Review. Cochrane Database Syst Rev. 2012;12(9) CD001901.

18. Marson A, Hutton J, Leach J, Castillo S, Schmidt D, White S, et al. Levetiracetam, oxcarbazepine, remacemide and zonisamide for drug resistant localizationrelated epilepsy: a systematic review. Epilepsy Res. 2001;46(3):259-270.

19. Koshy S, Thomas S, Nair C, Sarma P. Frequent seizures and polytherapy impair quality of life in persons with epilepsy. Neurol Asia. 2004:9(1):142.

20. Kumari P, Ram D, Nizamie SH, Goyal N. Stigma and quality of life in individuals with epilepsy: a preliminary report. Epilepsy Behav. 2009;15(3):358-361.

21. Sinha A, Sanyal D, Mallik S, Sengupta P, Dasgupta S. Factors associated with quality of life of patients with epilepsy attending a tertiary care hospital in Kolkata, India. Neurol Asia. 2011;16(1).

22. Basu S, Sanyal D, Ghosal M, Roy B, Senapati A, Das S. Psychometric properties of Bengali version of QOLIE-10 in epileptic patients. Ann Indian Acad Neurol. 2008;11(1):28. 